\title{
Prozess und Wirklichkeit
}

\section{Thomas Schweizer}

Dr. med., MAS Philosophie und Medizin, Mitglied Forum Medizin und Philosophie

Die zunehmenden, teilweise stupenden Möglichkeiten moderner Medizintechnik machen leicht vergessen, von welchen metaphysischen Voraussetzungen unsere Wissenschaft ausgeht. Wir fragen kaum mehr danach. Auch nicht, ob sie genügen, um das Wesen des Lebendigen zu verstehen. Das kann hier nicht umfassend diskutiert werden. Aber die Auseinandersetzung mit dem Begriff des Prozesses und seine Abgrenzung von dem uns geläufigeren der Funktion kann zu einer Überprüfung der gängigen Annahmen führen.

\section{Weg von den Dingen}

Vor rund hundert Jahren kam es zu grundlegenden Umwälzungen der Physik und damit den Grundlagen der Naturwissenschaften überhaupt. Materie und Energie liessen sich ineinander umwandeln. Ab diesem Moment schwenkte die Aufmerksamkeit der Physik weg von den Dingen (Substanzen) hin zu Prozessen, und in der Philosophie erkannte man, dass es nichts Seiendes gibt, das nicht ein Werdendes ist. Die Substanzontologie verwandelte sich in eine Prozessontologie. Es verdichten sich jetzt die Hinweise, dass sich das Prozessdenken von der Physik (und Philosophie) zu lösen und die Biologie massgebend zu prägen beginnt. Einmal in der Biologie angekommen, klopft es an die Tür der Medizin, und so sind wir Ärzte gehalten, uns mit Prozessen vertraut zu machen [1].

\section{Was ist ein Prozess?}

Das Verstehen von Prozessen hält manch eine unerwartete Schwierigkeit bereit. Wichtige Punkte seien hier stichwortartig genannt.

1. Gegebene Anfangsbedingungen bzw. das Gewordensein der Natur: Es ist objektivierbar und kann als eine Folge von Wirkursachen verstanden werden.

2. Ziele (auf welche sich ein Prozess hinbewegt) geistiger Art: Vorstellungen, Ideen etc., die den Erhalt des Lebens zum Inhalt haben. Dieser kann entgegen unseren bisherigen Annahmen durch die blinde Logik von Wirkketten nicht geleistet werden.

3. Eine auf das Ziel hinstrebende Energie als ein subjektiv erfahrenes Verlangen oder Begehren. Bereits einfachste Organismen kennen dieses Begehren. Bakterien, die sich in Richtung Nährstoffe bewegen, zieht es dorthin - Denken oder gar Sprache ist dazu nicht vonnöten. Es ist wichtig, sich das prozessuale Geschehen zuerst einmal als einen protomentalen Akt vorzustellen. Bewusstsein ist nicht Bedingung. Prozesse sind Erlebensakte, die in der Natur zumeist unbewusst ablaufen.

In den Punkten 2 und 3 unterscheidet sich der Prozess von demjenigen der Funktion, die mathematisierbare Abläufe beschreibt. Er entzieht sich zwangsläufig jeder Berechenbarkeit, weil ein kreatives Element ins Spiel kommt.

\section{Einfache und komplexe Prozesse}

Dieses kreative Moment erkennen wir, wenn bisweilen auch mit Mühe, in unserem Alltagsleben. Man kann den Prozess beschreiben als eine fortlaufende Realisierung von Zukunft, die zwar von den Bedingungen der Gegenwart ausgeht, aber gleichzeitig nach dem Nochnicht, nach dem Möglichen greift.

Antrieb jeglicher Prozesse aber ist das Erleben. Dieses kann nicht als Funktion beschrieben werden.

Organismen erleben, und jedes Erleben ist eine, wenn auch noch so kleine Verwandlung. Es schlummert darin etwas Zukunftsoffenes, Indeterminiertes. Beispiele eines Prozesses auf

- der chemischen Ebene: Die metabolischen Abläufe innerhalb eines Organismus sind Prozesse. Anders als dies beispielsweise bei Laborversuchen der Fall ist, nimmt ein lebendiger Organismus gleichzeitig und in einem sowohl den Standpunkt des Forschers wie auch denjenigen des Untersuchungsobjekts ein. Ein Organismus muss die im Labor statisch gegebenen Parameter intern als dynamische Variablen fortlaufend selber kontrollieren. Denn schon bei kleinsten Abweichungen verändert sich der metabolische Ablauf, und zwar meist ins Deletäre. Rein rechnerisch gehen nämlich die metabolischen Verläufe bei kleinsten Veränderungen sehr schnell weit auseinander. Die Wege des Lebendigen sind schmal. Das selbständige Reagieren und Regulieren ist ein Prozess, der nicht mittels mathematischer Funktionen zur Darstellung gebracht werden kann. 
- der mikrobiologischen Ebene: Wenn zwei biologisch identische Cyanobakterien-Kulturen, insbesondere auch mit identischem Phosphatgehalt, einer Sequenz von Phosphatstössen ausgesetzt werden, dann nehmen sie dieses Phosphat je nach ihrer Vergangenheit unterschiedlich schnell auf. Die Kolonie, deren Vorgängerkultur das Phosphat vorher in schnellen Stössen und im Übermass erhielt, tut es nun langsam, um sich so gegen die früher erfahrene Phosphatüberflutung zu schützen. Das setzt ein «Gedächtnis» und die Fähigkeit voraus, auf die Umgebung gestalterisch zu reagieren bzw. dem (im Übermass zerstörerischen) Phosphat etwas «entgegenzusetzen», was mathematisch wiederum nicht beschrieben werden kann [2]. Sie tun dies als ganz einfache Lebewesen, ohne dazu eine Sprache zu nutzen.

- Ebene Mensch: Ein technisch bis ins letzte determinierter chirurgischer Eingriff bedingt dennoch Wachsamkeit und Kreativität. Es ist 'diese Operation hier', die es so bisher noch nie gab. Ein sehr gutes Beispiel ist auch die Schaffung eines Kunstwerkes, das seinen Schöpfer überrascht und sogar verwandelt.

Die Natur ist ein Prozess, der dem Denken und der Sprache vorangeht. Das Momentum des Neuen, das diesem Geschehen innewohnt, ist schwierig zu fassen. Es ist das Resultat eines Suchens, das das Reale mit dem Potentiellen in Beziehung zu bringen vermag.

\section{Mögliche Konsequenzen für die Medizin}

Die möglichen Konsequenzen eines neuen, prozessorientierten Denkens in der Biologie und als Folge davon in der Medizin sind in ihrem Ausmass höchstens teilweise ausgelotet, können an dieser Stelle nur angedeutet werden und wären eine Diskussion in der Schweizerischen Ärztezeitung wert.

In der ärztlichen Praxis können wir die erhobenen Daten besser einordnen, wenn wir einen vagen Begriff der Geschichte der Patienten haben. Geschichten sind Prozesse. Wenn Prozesse harte Fakten z.B. in Form von Messdaten hervorbringen, dann sind diese Fakten Elemente oder Erscheinungen eines Geschehens, das sich nie vollständig fassen lässt.

Medizin - wir wissen es - ist nicht das Reagieren auf ein Faktum, sondern das Beeinflussen von Lebensvorgängen. Wir tun das seit jeher. Aber es macht einen Unterschied, ob man den 'Sternenhimmel' mit ptolemäischen oder mit kopernikanischen Augen betrachtet. Ob das mechanistische Denken übergeht in ein prozessuales. Denn dann werden wir das, was ge- schieht, als ein Narrativ verstehen. Eines, das sich fortlaufend weitererzählt. Teil des Narrativs sind auch wir, ist die Medizin, die Ärztin, der Arzt, die die Prozesse des Krankseins einerseits in rationale Gedanken übersetzen und Fakten rational lesen können, aber sie zugleich intuitiv erfassen. Erfassen heisst nicht sogleich erklären. Es ist eine Teilhabe am Erle-

\section{Es ist deshalb wichtig, dass wir die Messdaten nicht mit der Wirklichkeit verwechseln.}

ben. Empathie ist nicht ein Privileg des 'einfühlsamen' Arztes. Als Teilhabe am Prozess ist sie eine Bedingung für Erkenntnis.

\section{Forschung}

Wie soll man aber angesichts von ungenauen Prozessen genaue Forschung betreiben? Die rein datenbasierte Forschung übersieht in ihrer Suche nach Funktionen oft, dass Daten Abstrakta sind, die viel Konkretes ausblenden. Wesentlich ist oft das, was gar nicht abstrahiert werden kann. Die Daten sind Chiffren für sinnliche Ereignisse, die in einem ursprünglichen Kontext stehen, aber als 'brauchbare' Daten diesem oft entrissen und stattdessen in einen funktionalen Kontext gestellt sind, der die Prozesse des Lebendigen nur unvollständig erfasst.

\section{Hauptbotschaften}

1. Eine neue Biophilosophie wird sich nicht so sehr um Dinge (Substanzen) als vielmehr um Vorgänge (Prozesse) kümmern.

2. In Prozessen verschmelzen (a) wirkursächlich entstandene Anfangsbedingungen mit (b) Zielen und (c) dem subjektiv erlebten Verlangen (das Ziel zu erreichen) zu einer Einheit. Diese drei Elemente sind selbst in einfachsten Lebensvorgängen vorhanden und brauchen weder Sprache noch Selbstbewusstsein.

3. Eine prozessorientierte Medizin wird sich nicht mehr allein darauf konzentrieren, einen defekten Körper zu reparieren, sondern wird in einem gemeinsamen Prozess von Arzt und Patient Lebensvorgänge in eine günstige Richtung zu beeinflussen suchen.

\section{Literatur}

1 Der vorliegende Text basiert auf einem der Grundlagenwerke zum Thema "Prozessdenken»: Whitehead AN. Prozess und Wirklichkeit 1987 (1929) sowie auf neuen Arbeiten des Biophilosophen S. A. Koutroufinis: Organismus als Prozess (2019).

2 Falkner G und R. Die Selbstgestaltung der Lebewesen in Erfahrungsakten. Freiburg i. Br.: 2020. 